\title{
Edaravone combined with cinepazide maleate on neurocyte autophagy and neurological function in rats with subarachnoid hemorrhage
}

\author{
ZHENLI CAI $^{1}$, HAIDONG ZHANG ${ }^{1}$, HONGSHAN SONG ${ }^{1}$, YING PIAO $^{1}$ and XIAOYE ZHANG ${ }^{2}$ \\ ${ }^{1}$ Department of Neurology, Daqinglongnan Hospital, Daqing, Heilongjiang 163453; \\ ${ }^{2}$ Department of Oncology, Daqing People's Hospital, Daqing, Heilongjiang 163000, P.R. China
}

Received September 2, 2019; Accepted October 30, 2019

DOI: $10.3892 /$ etm. 2019.8240

\begin{abstract}
Effects of edaravone combined with cinepazide maleate on neurocyte autophagy and neurological function in rats with subarachnoid hemorrhage were investigated. Eighty SD rats were selected to establish subarachnoid hemorrhage (SAH) rat models, which were divided into sham operation group, SAH group, MCI group and combined group. Hippocampal tissue of each group was taken to observe the number of neurocytes. The expression levels of Beclin-1 and (light chain LC3)-II of rats in each group were detected by ELISA. Pearson's correlation factors were used to analyze the correlation between Beclin-1 and LC3-11, and neurological function tests were carried out on rats of each group 14 and 28 days after administration. The morphological and structural damage of nerve cells in the combined group was further alleviated, and the survival rate of neurons significantly increased at all time points $(\mathrm{P}<0.05)$. The expression levels of Beclin-1 and LC3-11 in combined group was significantly higher than those in SAH group and CMI group $(\mathrm{P}<0.05)$, and Beclin-1 was positively correlated with LC3-11 $(r=0.9454)$. Longa score of the combined group was significantly lower than that of the other two groups, and muscle strength score was significantly higher than that of the other two groups $(\mathrm{P}<0.05)$. Edaravone combined with cinepazide maleate can effectively increase the survival rate of brain cells and promote the volatilization of neurological function in the treatment of hemorrhage in the subretinal space of the omentum, which is worthy of popularization and application.
\end{abstract}

Correspondence to: Dr Zhenli Cai, Department of Neurology, Daqinglongnan Hospital, 35 Aiguo Road, Ranghulu, Daqing, Heilongjiang 163453, P.R. China

E-mail: cbdj66@163.com

Key words: subarachnoid hemorrhage, edaravone, cinepazide maleate, neurocyte autophagy, nerve function, combined therapy

\section{Introduction}

Subarachnoid hemorrhage (1) (SAH) refers to the rupture of diseased blood vessels at the bottom or surface of the brain. Blood directly flows into the subarachnoid cavity to cause a clinical syndrome, also known as primary subarachnoid hemorrhage, accounting for approximately $10 \%$ of acute stroke. It is a very serious and common disease. It is reported that secondary subarachnoid hemorrhage is caused by cerebral parenchyma, intraventricular hemorrhage, epidural or subdural vascular rupture, and vascular perforation of brain tissue into subarachnoid space (2).

Nimodipine used to be a common drug for the treatment of subarachnoid hemorrhage and the prevention of cerebral vasospasm, but clinical treatment found that this drug can increase intracranial pressure of patients, causing cerebral rebleeding, and cannot well protect brain tissue (3). Cinepazide maleate injection (CMI) (4) is a new type of drug with brain protection, which can effectively protect central nerve cells, expand cerebral vessels and improve brain microcirculation (5). Edaravone (6) is a free radical scavenger listed in 2001 . It can effectively regulate the expression of apoptosis genes, inhibit lipid peroxidation, prevent oxidative damage of the body, and play a role in vascular protection. It is widely used in the treatment of cerebral infarction, acute cerebral hemorrhage and other diseases (7).

Studies have found that autophagy participates in the pathological process of early brain injury (EBI) of subarachnoid hemorrhage and determines the outcome of nerve cells in EBI stage: recovery or repair, aggravation or death. At present, there is scarce research on the protective mechanism of edaravone and cinepazide maleate on $\operatorname{SAH}(8,9)$. Therefore, the SAH animal model was established in this study. Edaravone combined with cinepazide maleate was used for intervention, and the effects of expression and neurological function in key autophagy factor Beclin-1 and micro-related protein 1 (light chain LC3)-II were observed to analyze the protective mechanism of edaravone and cinepazide maleate on SAH nerve.

\section{Materials and methods}

Animal grouping. Eighty clean grade male SD rats weighing 350-450 g were purchased from Nanjing Junke Bioengineering 
Co., Ltd. They were divided into sham operation group, SAH group, CMI group and edaravone combined with CMI group (combined group). Each group was divided into four time subgroups of 24, 48, 72 and $144 \mathrm{~h}$.

The study was approved by the Ethics Committee of Daqinglongnan Hospital (Daqing, China).

Model making and treatment. The model of subarachnoid hemorrhage in rats was prepared by classic secondary blood injection method $(0.3 \mathrm{ml}$ of autologous arterial blood was injected into occipital cistern with a secondary interval of $48 \mathrm{~h}$ ). The sham operation group was injected by $0.3 \mathrm{ml}$ of normal saline into the occipital cistern twice, and the other operations were consistent with the SAH group. Criteria for model success: i) When secondary occipital cistern injection was performed, a small amount of hemorrhagic cerebrospinal fluid exuded from the puncture site; ii) when peeling off the brain, it was obvious that bloody liquid was scattered in the basal cistern of the brain floor. CMI group: $100 \mu \mathrm{g} / \mathrm{kg}$ was injected intraperitoneally immediately after SAH model was successfully prepared, and the drug was given once every $24 \mathrm{~h}$. The combined group received edaravone $10 \mathrm{mg} / \mathrm{kg}$ via tail vein on the basis of CMI, once every $24 \mathrm{~h}$.

Observation on morphology and structure of hippocampus in brain tissue. Two rats in each group were taken at each time point. After routine anesthesia treatment, the animals were sacrificed. After perfusion and fixation with $4 \%$ paraformaldehyde, paraffin embedding, coronal section, hematoxylin and eosin (H\&E) staining a 400-fold optical microscope was used to observe the morphological structure of hippocampal neurons. The number of viable nerve cells in each field of vision were counted, the specific method was as follows: Four slices of rats in hippocampus of each group were taken, 5 non-repeated fields of vision (100 fields in total) were selected from each slice, and Motic-6.0 image acquisition and analysis system were used for analysis, which was expressed by the ratio of the number of viable cells in CA1 area to the total number of cells under the average cell percentage (400x) in each field of vision.

ELISA detection of protein expression of related factors in rats. Blood was collected from vena cava, left in the greenhouse for $4 \mathrm{~h}$, centrifuged at $4^{\circ} \mathrm{C}$ and $3000 \mathrm{rpm}$ for $10 \mathrm{~min}$, and serum was collected and split-packed. ELISA kit was used to detect the relative expression levels of protein factors Beclin-1 and LC3-II. Finally, the absorbance (A) value of each sample at $450 \mathrm{~nm}$ wavelength was determined by using an enzyme-labeled instrument (to ensure that no water drops existed in the enzyme-labeled bottom plate and no bubbles were generated in the drop holes). After subtracting the A values of TMB blank color development holes from A values of all standard products and samples, the standard curve was drawn with the standard product concentration as the abscissa and the zero-adjusted A value as the ordinate, and the actual concentration of each sample was calculated. Beclin-1 ELISA kit was purchased from R\&D Systems, Inc. and LC3-II ELISA kit was purchased from Jianglai Biology.

Neurobehavioral scoring criteria and inclusion criteria. On the 14th and 28th day after administration, Longa nerve

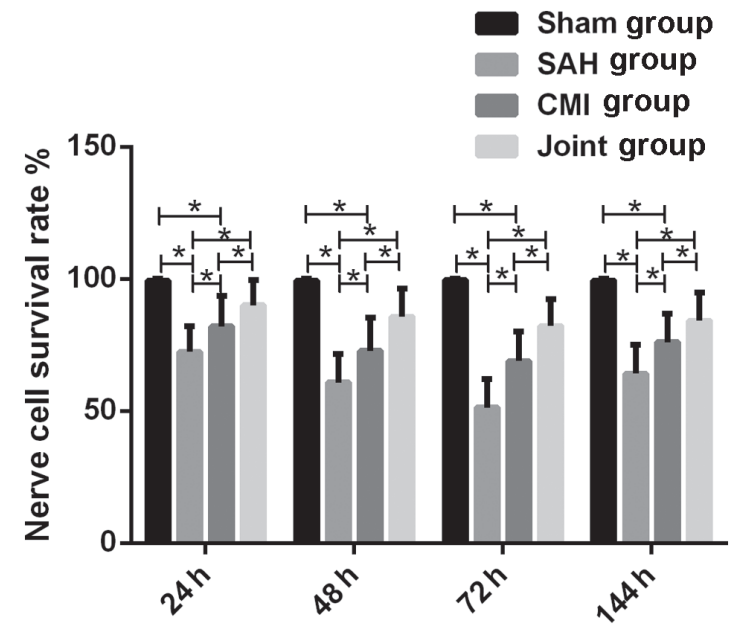

Figure 1. Comparison of the survival rate of nerve cells in hippocampus of each group. Compared with the sham operation group, the survival rate of nerve cells in SAH group, CMI group and combined group at various time points significantly reduced. The morphological and structural damage of nerve cells in CMI group was alleviated. Compared with SAH group, the number of viable nerve cells in visual field significantly increased. The morphological and structural damage of nerve cells in the combined group was further alleviated, and the survival rate of nerve cells significantly increased at all time points. ${ }^{*} \mathrm{P}<0.05$. SAH, subarachnoid hemorrhage; $\mathrm{CMI}$, cinepazide maleate injection.

function score and muscle strength score were respectively performed on rats in each group, and the scores were statistically analyzed.

Longa score (10). Neurological examination was divided into 5 grades: 0: normal, no neurological impairment; 1 point: left anterior paw could not be fully extended, with slight neurological impairment; 2 points: When walking, the rat turned to the left (paralyzed side) with moderate neurological deficits; 3 points: When walking, the body of the rat toppled to the left (paralyzed side), resulting in severe neurological deficits; 4 points: unable to walk spontaneously, loss of consciousness.

Muscle strength assessment (11). In order to reduce the error, we conducted two inspections and recorded the best results. The interval between the two inspections was $5 \mathrm{~min}$. The specific method was to add a diameter of 0 . A $6 \mathrm{~cm}$ nylon rope with a length of 1.0 meters was horizontally fixed at a height of $80.0 \mathrm{~cm}$, and a thick sponge was placed below to prevent rats from falling. Then, the tail was lifted and the forepaws of animals hang in the middle of the rope and the suspension time was recorded to indicate the strength of the forelimbs. The score was divided into 4 grades: 0 : hanging on the rope for $0-2$ sec; 1 point: hanging on the rope for $3-4 \mathrm{sec} ; 2$ points: hanging on the rope for more than $5 \mathrm{sec} ; 3$ points: hanging on the rope for more than $5 \mathrm{sec}$ and the hind legs could be put on the rope.

Statistical analysis. This study used SPSS18.0 (Bizinsight (Beijing) Information Technology Co., Ltd.) to carry out statistical analysis on the data. GraphPad Prism 6 software was used to draw all the pictures in this experiment. Chi-square test was used to compare the enumeration data, mean \pm standard deviation was used to express the measurement data, $\mathrm{T}$ test was used to analyze the two groups, variance analysis was 
A

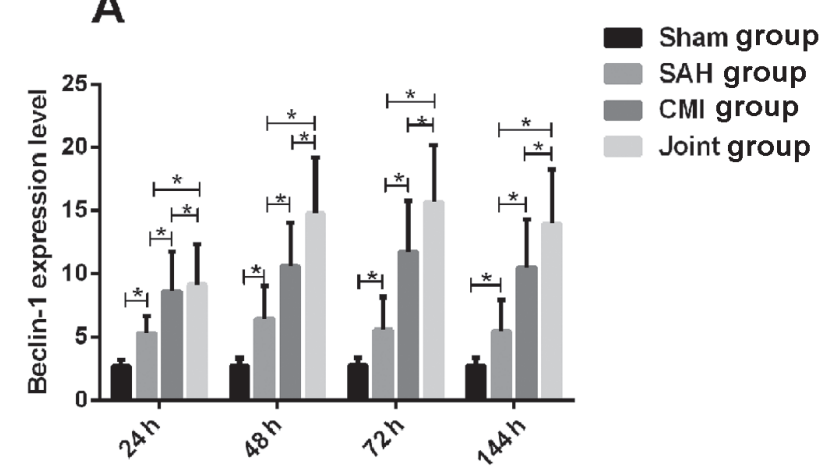

B

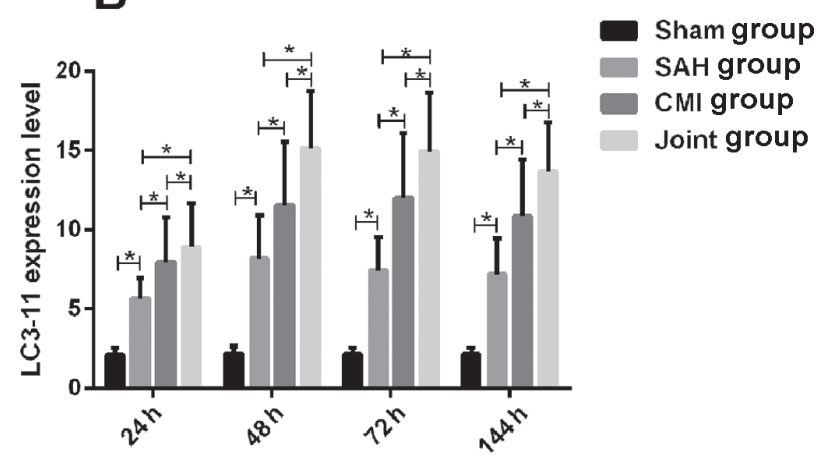

Figure 2. Expression of Beclin-1 and LC3-11 in rats of each group. (A) At each time point, the expression of Beclin-1 in combined group was significantly higher than that in the other three groups, and the expression of Beclin-1 in CMI group was significantly higher than that in SAH group. (B) At each time point, the expression of LC3-11 in combined group was significantly higher than that in the other three groups, and the expression of Beclin-1 in CMI group was significantly higher than that in SAH group. ${ }^{*} \mathrm{P}<0.05$. CMI, cinepazide maleate injection; $\mathrm{SAH}$, subarachnoid hemorrhage; LC, light chain.

used to compare the multiple groups, and Pearson's correlation analysis to analyze the relationship between variables. $\mathrm{P}<0.05$ was considered statistically significant.

\section{Results}

Comparison of survival rate of hippocampal nerve cells in each group. Compared with the sham operation group, the survival rate of nerve cells in SAH group, CMI group and combined group at each time point was significantly reduced $(\mathrm{P}<0.05)$. The morphological and structural damage of nerve cells in CMI group was alleviated. Compared with SAH group, the number of viable nerve cells in the visual field was significantly increased $(\mathrm{P}<0.05)$. The morphological and structural damage of nerve cells in the combined group was further alleviated, and their survival rate significantly increased at all time points $(\mathrm{P}<0.05)$ (Fig. 1).

Expression of Beclin-1 and LC3-11 in rats of each group. The expression levels of Beclin-1 and LC3-11 in SAH group was significantly higher than those in sham operation group $(\mathrm{P}<0.05)$. The expression levels of Beclin-1 and LC3-11 in $\mathrm{CMI}$ group was significantly higher than those in SAH group $(\mathrm{P}<0.05)$. The expression levels of Beclin-1 and LC3-11 in the combined group was significantly higher than those in $\mathrm{SAH}$ group and CMI group $(\mathrm{P}<0.05)$ (Fig. 2).

Correlation analysis between Beclin-1 and LC3-11. Pearson's correlation analysis showed that there was a correlation between Beclin-1 and LC3-11 ( $\mathrm{P}<0.05, \mathrm{r}=0.9454)$. The data of Beclin-1 and LC3-11 were taken from the index level measured in the Joint group for $72 \mathrm{~h}$ (Fig. 3).

Score of neurological function of rats in each group. Longa score and muscle strength score were performed at days 14 and 28 , respectively, of rats in the three groups. The results showed that Longa score in SAH group was significantly higher than CMI group and Joint group, while that in combined group was significantly lower than CMI group $(\mathrm{P}<0.05)$. The muscle strength score of SAH group was significantly lower than that of CMI group and Joint group, and the combined group was significantly higher than that of CMI group $(\mathrm{P}<0.05)$ (Fig. 4).

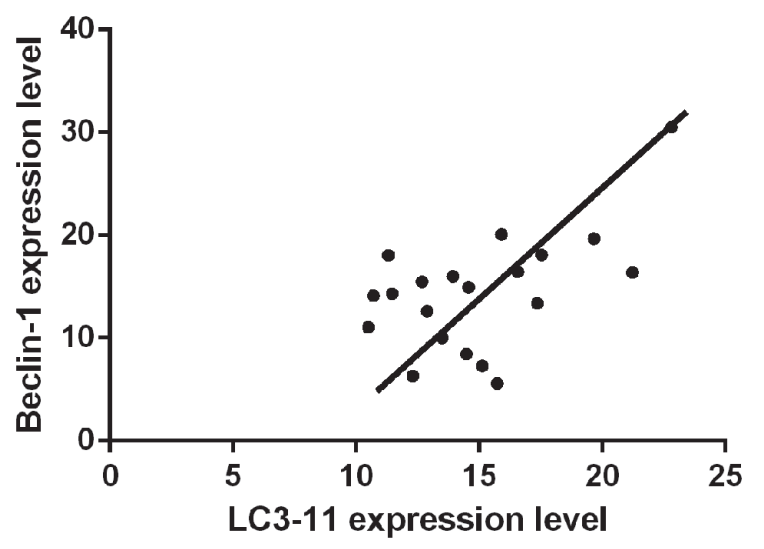

Figure 3. Correlation analysis between Beclin-1 and LC3-11. There was a positive correlation of Beclin-1 and LC3-11 ( $\mathrm{r}=0.9454, \mathrm{P}<0.05)$. LC, light chain.

\section{Discussion}

Subarachnoid hemorrhage is a common brain disease in clinical practice, and it is also a critical disease for life safety of crisis patients (12). The main causes of the disease are smoking, drinking, hypertension, trauma, hematological diseases, arterial angioma and other brain diseases (13). Patients often suffer from severe headache accompanied by dizziness, nausea, vomiting, pale complexion and cold sweat all over the body. With the aggravation of the disease, headache can radiate to the waist, back, neck and other parts, and even vascular spasm, cerebral infarction and limb hemiplegia in severe cases $(14,15)$. The key to treat the disease is to inhibit hemorrhage, prevent spasm and reduce the occurrence of complications. Cranial nerve autophagy is also an important reason for subarachnoid hemorrhage to harm cranial nerves.

It was found that CMI can reduce neuronal death in hippocampus of SAH rats and reduce neurological recovery disorders in animals, suggesting that CMI has a certain protective effect on EBI of SAH (16). Guidolzite maleate is a new type of ophthalmic calcium channel blocker, which can effectively prevent calcium ions from entering cells, relax vascular smooth muscle and dilate blood vessels, thus relieving 

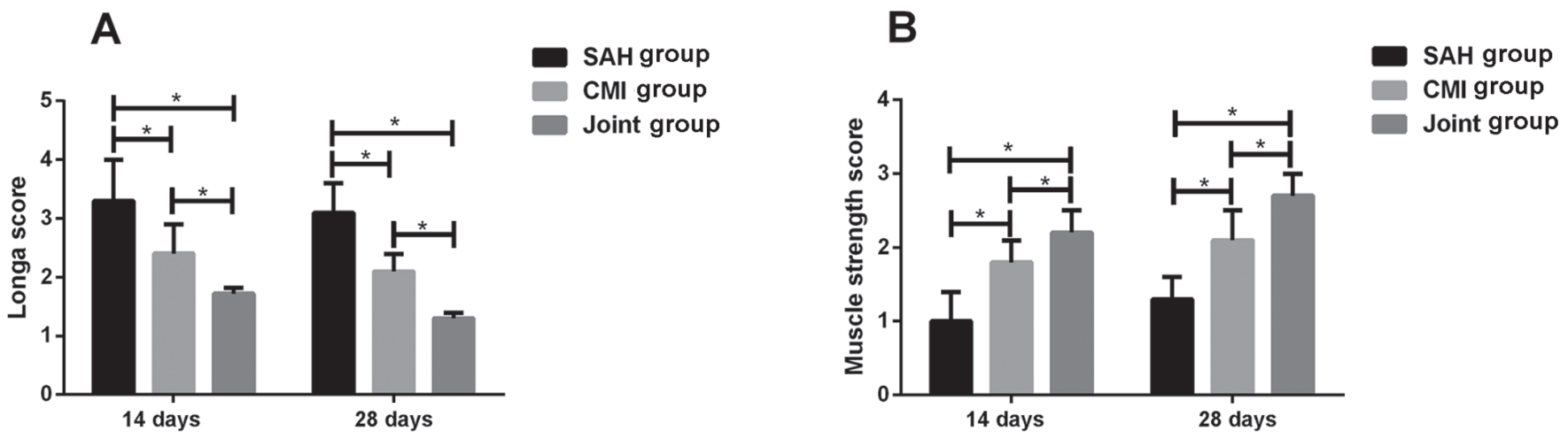

Figure 4. Score of neurological function of rats in each group. (A) At each time point, Longa score of SAH group was significantly higher than CMI group and combined group, Longa score of CMI group was significantly higher than combined group. (B) The muscle strength score of SAH group was significantly lower than that of CMI group and combined group, while that of CMI group was significantly lower than that of combined group. "P $<0.05$. SAH, subarachnoid hemorrhage; CMI, cinepazide maleate injection.

vascular healing twins, reducing blood flow resistance and increasing blood flow. It can also enhance the content of adenosine and adenosine cyclophosphosphate, reduce oxygen consumption, improve erythrocyte deformability, reduce blood stalk property, increase cerebral blood flow and improve cerebral metabolism (4). Previous studies have shown that CMI has a certain neuroprotective effect after treatment of ischemic encephalopathy $(17,18)$. In the state of brain injury, autophagy activation can clear damaged organelles in cells, regulate cell energy metabolism and reduce the degree of cell injury. However, excessive autophagy promotes caspase-3 lysis and aggravate the death of nerve cells (19). In this study, the expression levels of autophagy marker proteins Beclin-1 and LC3-11 in CMI group increased. At this time, the survival rate of nerve cells increased. On the one hand, it showed that autophagy activation in EBI stage of SAH had neuroprotective effect, and on the other hand, CMI played neuroprotective effect on SAH by enhancing autophagy degree in EBI stage.

It was found that edaravone had a good protective effect on SAH $(20,21)$. Intracranial blood accumulation after SAH, compression of coagulated blood clots, resulting in ischemia and anoxia, lysis of accumulated red blood cells, further release of superoxide anion and generation of hydroxyl radicals by oxyhemoglobin and increase of generation of oxygen radicals, induce strong oxidative stress reaction and aggravate nerve cell damage (22). In this study, Longa score and muscle strength score of the combined treatment group with added edaravone were significantly higher than those of CMI group and SAH group in neurological function score. Neuroprotective agent edaravone mainly prevents and treats the continuous progress of brain injury by scavenging oxygen free radicals and alleviating a series of cascade reactions caused by oxidative stress, which is the main reason for further improvement of the number of surviving nerve cells and nerve function in the combined group. The expression levels of Beclin-1 and LC3-11 in combined group increased further, suggesting that edaravone can strengthen CMI's regulation of autophagy activity after SAH. Edaravone can remove a large amount of oxygen free radicals, and the level of collective oxygen-free radicals is kept at a certain level, thus maintaining the moderate expression of autophagy level after SAH.
Collectively, edaravone combined with cinepazide maleate can effectively increase the survival rate of brain cells and promote the volatilization of nerve function in the treatment of hemorrhage in the subretinal space of the omentum, which is worthy of popularization and application.

\section{Acknowledgements}

Not applicable.

\section{Funding}

No funding was received.

\section{Availability of data and materials}

The datasets used and/or analyzed during the current study are available from the corresponding author on reasonable request.

\section{Authors' contributions}

$\mathrm{ZC}$ wrote the manuscript. ZC and $\mathrm{HZ}$ conceived and designed the study. ZC and HS were responsible for the collection and analysis of the experimental data. YP and XZ interpreted the data and drafted the manuscript. ZC and YP revised the manuscript critically for important intellectual content. All authors read and approved the final manuscript.

\section{Ethics approval and consent to participate}

The study was approved by the Ethics Committee of Daqinglongnan Hospital (Daqing, China).

\section{Patient consent for publication}

Not applicable.

\section{Conflict of interest}

The authors declare that they have no competing interests. 


\section{References}

1. Feigin VL, Rinkel GJ, Lawes CM, Algra A, Bennett DA, van Gijn J and Anderson CS: Risk factors for subarachnoid hemorrhage: An updated systematic review of epidemiological studies. Stroke 36: 2773-2780, 2005.

2. Locksley HB: Natural history of subarachnoid hemorrhage, intracranial aneurysms and arteriovenous malformations. Based on 6368 cases in the cooperative study. J Neurosurg 25: 219-239, 1966.

3. Kotwica $\mathrm{Z}$ and Persson L: Effect of intravenous infusion of nimodipine on intracranial pressure. Experimental study of ICP measurement conducted in a rat model. Neurol Neurochir Pol 23: 227-230, 1989 (In Polish).

4. Akashi A, Hirohashi M, Suzuki I, Shibamura S and Kasahara A: Cardiovascular pharmacology of cinepazide, a new cerebral vasodilator (author's transl). Nippon Yakurigaku Zasshi 75 507-516, 1979 (In Japanese).

5. Yi ZM, Liu F and Zhai SD: Cinepazide maleate injection for cerebral infarction: a systematic review. Chin J Evidence-Based Med 10: 1079-1084, 2010.

6. Kono H, Asakawa M, Fujii H, Maki A, Amemiya H, Yamamoto M, Matsuda M and Matsumoto Y: Edaravone, a novel free radical scavenger, prevents liver injury and mortality in rats administered endotoxin. J Pharmacol Exp Ther 307: 74-82, 2003.

7. Jin Q, Cai Y, Li S, Liu H, Zhou X, Lu C, Gao X, Qian J, Zhang J, $\mathrm{Ju} \mathrm{S}$ and Li C: Edaravone-encapsulated agonistic micelles rescue ischemic brain tissue by tuning blood-brain barrier permeability. Theranostics 7: 884-898, 2017.

8. Wang Z, Shi XY, Yin J, Zuo G, Zhang J and Chen G: Role of autophagy in early brain injury after experimental subarachnoid hemorrhage. J Mol Neurosci 46: 192-202, 2012.

9. Lee JY, He Y, Sagher O, Keep R, Hua Y and Xi G: Activated autophagy pathway in experimental subarachnoid hemorrhage. Brain Res 1287: 126-135, 2009.

10. Zheng XW, Yang WT, Chen S, Xu QQ, Shan CS, Zheng GQ and Ruan JC: Neuroprotection of catalpol for experimental acute focal ischemic stroke: Preclinical evidence and possible mechanisms of antioxidation, anti-inflammation, and antiapoptosis. Oxid Med Cell Longev 2017: 5058609, 2017.

11. Lu XY and Hao XM: Biological effects of electrical stimulation on muscle strength and electromyogram in rats with muscle disuse atrophy. Chin J Clin Rehabil 10: 34-36, 2006 (In Chinese).

12. McGirt MJ, Mavropoulos JC, McGirt LY, Alexander MJ, Friedman AH, Laskowitz DT and Lynch JR: Leukocytosis as an independent risk factor for cerebral vasospasm following aneurysmal subarachnoid hemorrhage. J Neurosurg 98: 1222-1226, 2003.
13. Hillbom $\mathrm{M}$ and Kaste $\mathrm{M}$ : Alcohol intoxication: A risk factor for primary subarachnoid hemorrhage. Neurology 32: 706-711, 1982.

14. Yolas C, Ozdemir NG, Kanat A, Aydin MD, Keles P, Kepoglu U, Aydin N and Gundogdu C: Uncovering a new cause of obstructive hydrocephalus following subarachnoid hemorrhage: choroidal artery vasospasm-related ependymal cell degeneration and aqueductal stenosis-first experimental study. World Neurosurg 90: 484-491, 2016

15. Hartmann A, Dorndorf W and Alberti E: Complications of subarachnoid hemorrhage. Med Klin 72: 476-482, 1977.

16. Lee JY, Sagher O, Keep R, Hua Y and Xi G: Comparison of experimental rat models of early brain injury after subarachnoid hemorrhage. Neurosurgery 65: 331-343, 2009.

17. Chao Y, Hu W and Geng X: The neuroprotective strategic analysis for patients with acute myocardial infarction combined with hypoxic ischemic encephalopathy in ICU. Hellenic J Cardiol 58: 427-431, 2017.

18. Zheng PU, Wang XR, Gan J and Chen QI: Effect of cinepazide maleate preconditioning on rat models of cerebral ischemia. J Shanghai Jiaotong Univ 33: 1064-1073, 2013 (In Chinese).

19. Wang L, Wang P, Dong H, Wang S, Chu H, Yan W and Zhang X: U1k1/FUNDC1 prevents nerve cells from hypoxia-induced apoptosis by promoting cell autophagy. Neurochem Res 43: 1539-1548, 2018.

20. Munakata A, Ohkuma H, Nakano T, Shimamura N, Asano K and Naraoka M: Effect of a free radical scavenger, edaravone, in the treatment of patients with aneurysmal subarachnoid hemorrhage. Neurosurgery 64: 423-429, 2009

21. Wei W, Yang G, Shu XU, Yin W-B and Ding X-S: The brainprotective effects of edaravone in rats after subarachnoid hemorrhage. Chin J Contemp Neurol Neurosurg 8: 544-549, 2008 (In Chinese).

22. Nakagomi T, Yamakawa K, Sasaki T, Saito I and Takakura K: Effect of edaravone on cerebral vasospasm following experimental subarachnoid hemorrhage. J Stroke Cerebrovasc Dis 12: $17-21,2003$

This work is licensed under a Creative Common Attribution-NonCommercial-NoDerivatives 4.0 International (CC BY-NC-ND 4.0) License. 